\title{
Femoral Hernia: Epidemiology and challenges of management in a sub-saharan African Country
}

\author{
Aloysius Ugwu-Olisa Ogbuanya ${ }^{1,2,3,4}$, Fabian Ugonna Olisa ${ }^{1}$, Richard Lawrence Ewah $^{2,5,6}$, \\ Obinna Nweke ${ }^{7}$, Nonyelum Benedett Ugwu ${ }^{8}$ \\ ${ }^{1}$ Consultant Surgeon, Department of Surgery, Alex Ekwueme Federal University Teaching Hospital, Abalaliki \\ (AEFUTHA), PMB 102, Ebonyi State, Nigeria, '2Lecturer, Department of Surgery, Ebonyi State University, Abakaliki \\ (EBSU), Ebonyi State, Nigeria, ${ }^{3}$ Consultant Surgeon, Department of Surgery, Bishop Shanahan Specialist Hospital, \\ Nsukka, Enugu State, Nigeria, ${ }^{4}$ Consultant Surgeon, Department of Surgery, Mater Misericordiae Hospital, Afikpo, \\ Ebonyi State, Nigeria, ${ }^{5}$ Consultant Anaesthetist, Department of Anaesthesia, Alex Ekwueme Federal University \\ Teaching Hospital, Abalaliki (AEFUTHA), PMB 102, Ebonyi State, Nigeria, ${ }^{6}$ Consultant Anaesthetist, Department of \\ Anaesthesia, Mater Misericordiae Hospital, Afikpo, Ebonyi State, Nigeria, ${ }^{7}$ Senior Resient, Department of Surgery, \\ Alex Ekwueme Federal University Teaching Hospital, Abalaliki (AEFUTHA), PMB 102, Ebonyi State, Nigeria, ${ }^{8}$ Nursing \\ Officer, Department of Nursing, Bishop Shanahan Specialist Hospital, Nsukka
}

Background: Femoral hernia is peculiar due to its high propensity towards strangulation, high rates of diagnostic pitfalls and occurrence in older patients with multiple comorbidities. Therefore, an updated record of the clinical characteristics and burden of the disease in our locality should be established. Aims and Objectives: This study aimed to document the challenges of diagnosis and outcome of repair of femoral hernia in southeast Nigeria. Materials and Methods: This was a multi-centre retrospective study of adult patients with femoral hernias repaired surgically over twelveyears period. Tests of statistical significances were done. Results: A total of 128 patients were studied and they represented $3.4 \%$ and $2.6 \%$ of all patients with groin and abdominal wall hernias respectively. There were 116 females and 12 males. The overall rate of missed diagnosis was $14.8 \%$; majority (84.2\%) were in the emergency arm. Eighty-one (63.4\%) patients presented emergently while 47 $(36.7 \%)$ were managed electively. In the emergency group, the vast majority $(72,88.9 \%)$ presented beyond 24hours from onset of complications. Still on the emergency group, 72 $(88.9 \%)$ were strangulated, seven $(8.6 \%)$ were obstructed and $2(2.5 \%)$ were incarcerated at presentation. Overall resection rate was $39.8 \%$, but in the emergency group, it was $63.0 \%$. Prosthetic meshes were used in only $4(8.5 \%)$ of the 47 elective cases. Recurrence rate was Overall morbidity and mortality rates were $43.0 \%$ and $10.9 \%$ respectively. Late presentation was an independent predictor of mortality $(p=0.000)$. Conclusion: Thoughfemoral hernias are relatively uncommon, the rate of missed diagnosis is high, majority of which occur in the emergency group. Majority presented in emergency and have high propensity towards strangulation with attendant high morbidity and mortality.

Key words: Femoral hernia; Mesh plug; Strangulation; Mortality; Resection
Access this article online

Website:

http://nepjol.info/index.php/AJMS

DOI: 10.3126/ajms.v11i6.30368

E-ISSN: 2091-0576

P-ISSN: 2467-9100

Copyright (c) 2020 Asian Journal of Medical Sciences

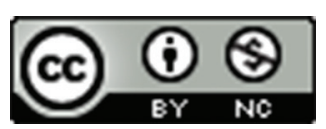

This work is licensed under a Creative Commons Attribution-NonCommercial 4.0 International License.

\section{INTRODUCTION}

Femoral hernias are relatively uncommon, making up $2-8 \%$ of all adult groin hernias and less than $5 \%$ of all abdominal wall hernias. ${ }^{1,2,3}$ About $60 \%$ of femoral hernias are on the right, $30 \%$ on the left and 10\% bilaterally. ${ }^{3,4}$ Femoral hernias are very rare in children, occur commonly in the 40-70years age group with a female preponderance in excess of four to five folds, especially in multiparous women. ${ }^{3}$ However, a woman with a groin mass is still five times more likely to have an inguinal hernia than a femoral hernia. ${ }^{5}$ Nevertheless, the major problem with femoral hernia does not lie with its frequency 
among abdominal wall hernias, rather on its high propensity towards strangulation, high rates of diagnostic pitfalls and occurrence in older patients with multiple comorbidities. ${ }^{5,6}$

Indeed, femoral hernia is the most commonly incarcerated abdominal wall hernia; approximately $40 \%$ of femoral hernias manifest with strangulation and post-operative complication rates have been reported to be over 50\% in patients with intestinal resection. ${ }^{1,5-10}$ Because femoral hernias are less frequently encountered in general surgical practice than other types of primary groin hernias or midline ventral hernias, the experiences reported by most researchers were encapsulated in case reports and case series. ${ }^{6,11-14}$ In Turkey, $27(51.9 \%)$ of 52 patients evaluated for femoral herniapresented in emergency and $85 \%$ of the emergency cases had small bowel resection. ${ }^{9}$ In the emergency group still, post-operative complications occurred in $27 \%$, characterized by major morbidities like anastomotic leakage and pulmonary complications ${ }^{9}$. Unfortunately, reports culled from various regions in Africa, India and Europe agree uniformly with the Turkish results. ${ }^{7,15-18}$

In Nigeria, particularly in our environment, data on femoral hernia are disturbingly limited despite the infrequent, but continuous clinical encounter with this hernia. The morbidity and mortality toll that accompany strangulated femoral hernia and the persistent clinical trap associated with its diagnosis constitute a major indication why an updated record of the clinical characteristics andburden of the disease in our own locality should be established. This study aimed to document the challenges of diagnosis and outcome of repair of femoral hernia in south east Nigeria.

\section{MATERIALS AND METHODS}

\section{Design and setting}

Thiswas a retrospective study of consecutive patients with femoral hernias repaired from January, 2007 to December, 2018. All the patients were managed at the three hospitals.

\section{Subjects}

The case folders of the patients with clinical diagnosis of abdominal wall hernia was initially retrieves from the records departments of the three hospitals and their specific diagnoses recorded. Those with diagnoses of femoralhernias were selected for further evaluation. Those with incomplete clinical details were excluded. Details of their socio-demographic characteristics, mode of presentation, duration of hernia before presentation, contents of the sacs, comorbid conditions, method of repair, anesthetic techniques and treatment outcomes, including recurrence and mortality were obtained and recorded. For the complicated cases, the degree of bowel involvement (obstruction, strangulation, gangrene) and bowel resection rates were noted and recorded. The post-operative outcomes during follow up of the patients were recorded.

\section{Data analysis}

Data analysis was done using Statistical Package for Social Sciences (SPSS) software version 22.0 (IBM, Chicago, IL USA). Data were presented as means, tables, figures, percentages and standard deviation. Confidence interval was calculated at 95\% level and significance at 5\% probability level $(\mathrm{p}<0.05)$.

\section{Ethical approval}

The proposal for this study was approved by the research and ethics committee of the hospitals before commencement of the study. All research principles relating to retrospective studies on human subjects were adhered to during the study.

\section{RESULTS}

\section{Socio-demographic characteristics}

During the period under review, 128 patients had repairs for femoral hernia and they comprised 3.4\% of 3,765 patients with groin hernia and $2.6 \%$ of 4,839 patients with abdominal wall hernia. Therefore, patients with groin hernias represented $77.8 \%$ of all patients with abdominal wall hernia in our environment during the study period. The 128 patients with femoral hernia formed our study population. Of the patients with femoral hernia, there were 116 females and 12 males giving male to female ratio of 1:9.7. There was statistically significant difference in the sex of the patients $(p=0.001)$. The ages of the patients ranged from 16-92 years with a mean of $50.81+/-$ SD 18.11. Majority $(77,60.2 \%)$ were farmers, followed by artisans $(24,18.8 \%)$, traders $(12$, $9.4 \%)$, laborers $(9,7.0 \%)$, professionals $(3,2.3 \%)$ and others $(3,2.3 \%)$. Majority $(106,82.8 \%)$ were rural dwellers; the rest were either semi-urban or urban $(22,17.2 \%)$ dwellers.

\section{Clinical presentation}

One hundred and twenty-seven patients had unilateral hernia; only one patient harbored bilateral hernia giving rise to a total of 129 hernias. The single patient with bilateral hernia had no complications in both groins. There were $19(14.8 \%)$ patients with recurrent hernias; 16 emergency cases and three electives (Table 1).

Late presentation was common.Eighty-one (63.3\%) patients presented emergently while 47 (36.7\%) had elective presentation. At the time of first contact in the hospital, missed diagnosis occurred initially in the course of clinical assessment in $16(19.8 \%)$ patients with emergency presentation and in three $(6.4 \%)$ patients with elective cases. The overall rate of missed diagnosis was $14.8 \%$. But correct diagnoses were made in subsequent assessments.Forty-nine 
$(38.3 \%)$ patients agreed that they had lived with the hernias for more than five years while $29(22.7 \%)$ said they were not aware of any pre-existing groin hernia prior to presentation. Eleven $(8.6 \%)$ patients harbored the hernias for more than 25 years before presentation, but only 10 (7.8\%) patients gave a short history of one year or less. For the 81 patients with emergency presentation, only nine $(11.1 \%)$ presented within 24 hours after complications started. The relationship between delay in presentation after onset of complications and degree of bowel involvement is shown below (Table 2).

The other peri-operative findings are shown below (Table 3). Fifty-two (40.6\%) patients had comorbidities. Some patients had two or more comorbidities giving a total of 72 comorbid conditions ranging from hypertension (33, $45.8 \%$ ), diabetes mellitus $(13,18.1 \%)$, obesity $(10,13.9 \%)$, Chronic obstructive pulmonary disease (6, 8.3\%), benign prostatic hyperplasia (4, 5.6\%), HIV/AIDS (2, 2.8\%), Chronic renal disease $(2,2.8 \%)$ and goiter $(2,2.8 \%)$.

Surgical treatment and anesthetic assessments Fifty one of 81 patients that presented in emergency had intestinal resection, giving a resection rate of $39.8 \%$ for

\begin{tabular}{lcccc}
\multicolumn{5}{l}{ Table 1: Clinical characteristics of the hernias } \\
\hline Parameter & Emergency & Elective & Total & Percent (\%) \\
\hline Age (years) & & & & \\
$16-45$ & 17 & 25 & 42 & 32.8 \\
$46-65$ & 35 & $17^{*}$ & 52 & 40.6 \\
$66-87$ & 29 & 5 & 34 & 23.3 \\
$\quad$ Total & 81 & 47 & 128 & 100.0 \\
Sidedness & & & & \\
$\quad$ Right & 57 & 31 & 88 & 68.8 \\
Left & 24 & 15 & 39 & 30.5 \\
Bilateral & 0 & 1 & 1 & 0.7 \\
$\quad$ Total & 81 & 47 & 128 & 100.0 \\
Type of hernia & & & & \\
$\quad$ Primary & 65 & $44^{*}$ & 109 & 85.2 \\
Recurrent & 16 & 3 & 19 & 14.8 \\
$\quad$ Total & 81 & 47 & 128 & 100.0 \\
Gender & & & & \\
$\quad$ Male & 4 & 8 & 12 & 904 \\
Female & 77 & 39 & 116 & 90.6 \\
$\quad$ Total & 81 & 47 & 128 & 100.0 \\
Diagnostic challenge & & & & \\
$\quad$ Prompt diagnosis & 65 & 44 & 109 & 85.2 \\
Missed diagnosis & 16 & 3 & 19 & 14.8 \\
Total & 81 & 47 & 128 & 100.0 \\
\hline *Single patient with bilateral hernia belongs to this group. & \\
$\quad$ & & &
\end{tabular}

entire patients with femoral hernia and $63.0 \%$ for emergency presentation.Majority $(84,65.6 \%)$ of the surgical repairs were done under general anesthesia, followed by spinal anesthesia $(24,18.8 \%)$. There was statistically significant difference in the number of emergency cases performed under emergency and spinal anaesthesia compared to elective cases $(p=0.031)$. The effects of American Association of Anesthesiologists (ASA) class on the choice of anesthetic technique are shown below (Table 4).Midline laparotomy incisions were used in 79 (77.5\%) of 81 patients that underwent emergency operations; the remaining two cases of emergency operation had incarcerated omentum and after resuscitation, Lockwood (low approach) approach was utilized for the repair. The remaining 47 patients with elective repair, had their repairs through the low (Lockwood) approach.

\section{Outcomes of treatment}

Fifty-five $(43.0 \%)$ patients had post-operative complications;39 $(70.9 \%)$ were in the emergency group and $16(29.1 \%)$ in the elective group $(p=0.026)$. Some had two or more postoperativeevents giving rise to a total of 74 complications. The complicationrates included wound infection $(40,31.3 \%)$, anastomotic leak $(5,3.9 \%)$, intraperitoneal sepsis $(6,4.6 \%)$, recurrence $(7,5.5 \%)$ and others $(3,2.3 \%)$. Intestinal resection was an independent predictor of wound infection $(\mathrm{p}=002)$. All the bowel resections (51, $100.0 \%)$ and deaths $(14,100.0 \%)$ occurred in those who presented acutely and in those admitted after 24 hours following onset of complications (Table 5). Late presentation was an independent predictor of bowel resection $(p=0.000)$ and mortality $(p=0.000)$. The overall mortality rate was $10.9 \%$ (14 deaths), but in the emergency group, it was $17.3 \%$. Five $(71.4 \%)$ of the seven recurrences were in the emergency group, the remaining two $(28.6 \%)$ occurred in the elective group. Postoperative wound infection significantly contributed to recurrence $(\mathrm{p}=0.010)$. From hospitals' records, duration of follow up ranged from 2-18 months with a median of 10 months.

\section{DISCUSSION}

In order to document and analyze the natural history of an uncommon surgical disease like femoral hernia, a multicenter study aimed at obtaining a pool of unselected cohorts is warranted.Therefore, a report on the cases of femoral

\section{Table 2: Duration of delay after onset of complication}

\begin{tabular}{lccccc}
\hline Delay (hours) & Incarcerated & Obstruction & Strangulation & Total (\%) & P-value \\
\hline $0-24$ & 0 & 5 & 4 & $9(11.1)$ & $11(13.6)$ \\
$25-48$ & 0 & 0 & 11 & $19(23.5)$ \\
$49-72$ & 0 & 1 & 18 & $28(34.6)$ \\
$>72$ & 0 & 1 & 27 & $14(17.3)$ & \\
Not stated & 2 & 0 & 12 & $81(100.0)$ \\
Total (\%) & $2(2.5)$ & $7(8.6)$ & $72(88.9)$ & &
\end{tabular}

$\dagger>=$ greater than 
hernias managed in tertiary and rural-based mission hospitals was undertaken to present the flavor of experiences gained by surgeons with both central and district hospital practice. The high rates $(19.8 \%$ for emergency and 6.4\% for elective cases) of missed diagnosis during the time of evaluation of patients with femoral hernia in this series and the accompanying persistent clinical trap is worrisome and may partly explain the relative rarity and paucity of original articles on femoral hernias reported in the surgical literatures. ${ }^{19}$

The major trouble with femoral hernia is therefore two-pronged; first, delayed presentation due to missed diagnosis and second, the propensity towards strangulation, commonly in older patients. ${ }^{1,3,19}$ Perhaps, this observation may partly explain the high rate of emergency presentation and strangulated cases recorded in this series. The high rate of complicated femoral hernia (63.3\%) observed in this study conformed to rate of $63.2 \%$ in Japan and comparable to a figure of $83.3 \%$ in India. ${ }^{17,20,21}$ Elsewhere, the early involvement of intestinal segment in complicated femoral hernia sacs has been attributed to narrow femoral canal and tight femoral ring. ${ }^{20}$ From the above, it can be deduced that femoral hernias incarcerate and become strangulated readily in all races, regardless of the health seeking behavior of the population, probably due to its persistent clinical obscurity, especially in obese patients as well as the tight femoral ring and narrow femoral canal.

\begin{tabular}{lcccc}
\hline \multicolumn{5}{l}{ Table 3: Peri-operative characteristics } \\
\hline Clinical parameter & Elective & Emergency & Total & Percent (\%) \\
\hline Content of hernia sac & & & & \\
Small bowel & $4^{*}$ & 79 & 83 & 64.8 \\
Omentum & 23 & 2 & 25 & 19.5 \\
Empty & 9 & 0 & 9 & 7.1 \\
Others & 1 & 0 & 1 & 0.8 \\
Not stated & 10 & 0 & 10 & 7.8 \\
Total & 47 & 81 & 128 & 100.0 \\
Bowel resection & & & & \\
Yes & 0 & 51 & 51 & 39.8 \\
No resection & 47 & 30 & 77 & 60.2 \\
Total & 47 & 81 & 128 & 100.0 \\
Type of repair & & & & \\
Suture-based & 43 & 81 & 124 & 96.9 \\
Prosthetic & 4 & 0 & 4 & 31.0 \\
Total & 47 & 81 & 128 & 100.0 \\
\hline * Single patient with bilateral hernia belongs here. & & \\
\hline
\end{tabular}

In this review, $67.2 \%$ of the entire patients were aged 46years and above while less than a tenth (12, 9.4\%) of all the patients with femoral hernias were males; both findings highlight the predilection of femoral hernia for female sex and ageing patients, as previously observed by other authors. ${ }^{2,3,9,10,17,21}$ The right-sided preponderance noted in this series uniformly agrees with reports culled from India, Toronto, and Republic of Serbia. ${ }^{8,10,17}$ The explanation for the greater incidence of femoral hernias in women is the increased tendency of femoral canal to be larger and oval shaped in women compared to men while the anatomic position of the sigmoid colon which creates a tamponade on the left femoral canal has been suggested to be the cause of lower frequency of femoral hernias on the left side. $8,9,17,21$ The occurrence of pregnancy in women is an additional predisposing factor and further contributes to higher incidence of femoral hernias in females. ${ }^{2,17,18}$

The relative rarity of femoral hernia in children and young adults as noted in this study has been highlighted by other authors. ${ }^{2,3,21}$ The theory of congenital preformed pre-peritoneal sac has been discredited and the weight of evidence is currently laid on the acquired theory and this may partly explain why femoral hernias are more common in middle aged and elderly patients. ${ }^{3}$

The rate of bilateral femoral hernia in this study is rather low $(0.8 \%)$ compared to rates of $3.0-6.3 \%$ in Europe. ${ }^{3,8,10,16}$ It is far lower than value documented for inguinal hernia in our institution (17.2\%) and Tanzania (20.4\%). ${ }^{22,23}$ In most series in Africa and India, no case of bilateral femoral hernia was reported despite relatively large number of the study cohorts in some of the reports. ${ }^{2,17,18}$ It appears bilateral femoral hernias are more commonin the western nations than developing countries for reasons yet to be discovered. Nevertheless, femoral hernias were thought to be more common among the whites generally, and the fact that in Africa, people walk barefoot contributes to significant inguinal lymphadenitis leading to obliteration of femoral canal and subsequent lower incidence of femoral hernias in blacks. ${ }^{7}$

Patients with recurrent hernias were many (14.1\%), probably due to the less effective anatomic repair technique used in the previous operations. All the 16 patients who presented with

Table 4: Effects of ASA classification on anesthetic technique and mortality rate

\begin{tabular}{lccccc}
\hline Anesthetic method & ASA I-II & ASA III & ASA IV & Total (\%) & P-value \\
\hline Local & 19 & 0 & 0 & $19(12.5)$ & 0.031 \\
Local + sedative & 1 & 1 & 5 & $23(18.1)$ & \\
Spinal & 14 & 7 & 2 & $79(65.6)$ & \\
General & 10 & 64 & $12(9.4)$ & $128(100.0)$ & 0.000 \\
Total (\%) & $44(34.4)$ & $72(56.2)$ & $5(41.7 \%)$ & $14(10.9 \%)$ & \\
Mortality (\%) & $0(0.0 \%)$ & $9(12.5 \%)$ & &
\end{tabular}




\begin{tabular}{|c|c|c|c|}
\hline Delay (hours) & $\begin{array}{c}\text { Number of Resection } \\
(\%)\end{array}$ & $\begin{array}{l}\text { Morbidity } \\
\text { (\%) }\end{array}$ & $\begin{array}{c}\text { Mortality } \\
(\%)\end{array}$ \\
\hline $0-240$ & $(0.0)$ & $1(11.1)$ & $0(0.0)$ \\
\hline $25-48$ & $2(18.2)$ & $3(27.3)$ & $1(9.1)$ \\
\hline $49-72$ & $15(78.9)$ & $10(52.6)$ & $3(15.8)$ \\
\hline$>72$ & $24(85.7)$ & 19 (67.9) & $8(28.6)$ \\
\hline Not stated & $10(71.4)$ & $6(42.9)$ & $2(14.3)$ \\
\hline Total & $51(63.0)$ & $39(48.1)$ & $14(17.3)$ \\
\hline P-value & 0.000 & 0.031 & 0.000 \\
\hline
\end{tabular}

hernia recurrencesat first contact had their previous repairs outside a teaching hospital and the hernias were repaired by general duty doctors who have inadequate experience and insufficient surgical skills to undertake such repairs. In addition, the low utilization of prosthetic implants for hernia repairs among many surgeons in our environment cannot be overlooked and such reasons like no availability of mesh, unaffordability of mesh, ignorance, socio-cultural practices and lack of experience in mesh implantation have been found to contribute to low application of prosthetic meshes in groin hernia in our environment or across many African and other developing nations. ${ }^{22}$ In the pre-mesh era, Frank from Shouldice hospital, Toronto, found that $23.7 \%$ (91 cases) of the 384 femoral herniorrhaphies performed in his institution were due to recurrent hernias. ${ }^{10}$ Unfortunately,the above results in the pre-mesh period in the West are still reported in the contemporary time in our practice, ${ }^{23}$ and in other centers in Uganda ${ }^{18}$ and Nigeria, ${ }^{13}$ emphasizing the much celebrated view that repair by suture-based methods is still popular in Africa, which probably contributed to the high recurrences in the previously repaired hernias that were encountered in this study. In the current review, only $4(8.5 \%)$ of the 47 patients that presented electively had prosthetic mesh repair, further attesting to the low utilization of meshes for femoral hernia repair in our environment. The overall recurrence rate of $5.5 \%$ observed in the current series is still worrisome considering the short follow up period, and high emergency rate precluding mesh implants as well as low uptake of mesh in the elective group may have contributed to the high early repair failure rates.

Curiously, Dauda and Colleague from northern Nigeria reported on a single case of longstanding giant femoral hernia in a 52year old male and repaired the hernia without mesh implants despite the large volume of the hernia and absence of strangulation. ${ }^{13}$ The authors also observed that the giant hernia was irreducible for over one year without strangulation. The investigators cited that though, femoral herniasare more common in females, the giant forms are more common in malesand that the irreducible longstanding types have low incidence of strangulation. ${ }^{13}$ However, the fact that the study was a case report created significant limitation for the above work and calls for cautious attitude while making observations of robust clinical importance using a case report.

The foregoing illustrates the low popularity of prosthetic implants for femoral hernia repair in our environment probably due to reasons mentioned previously and low surgical output of femoral hernia that has limited the experiences with mesh. However, in Cairo, Egypt, Mohammed used a mesh plug to repair all the 23 uncomplicated femoral hernias treated in his series and reported no recurrence and a single case each for wound infection, mild pain and seroma. ${ }^{2}$ The author recommended the use of polypropylene mesh plug through an infrainguinal approach for satisfactory closure of the femoral canal akin to reports from other similar studies. ${ }^{8,9}$

Investigators from Japan have moved steps forward, and recommended use of mesh implants for incarcerated and strangulated femoral hernia repair provided there is no contamination of the operation field. ${ }^{21}$ In a review involving 24 patients that underwent emergent femoral hernia repair, it was reported that 23 had bowel resection, and of these, 15 $(65.2 \%)$ had their hernias repaired with mesh implants. ${ }^{21}$ The authors recorded only two cases of wound infection, one peritonitis requiring re-operation and no recurrences after a follow-up period ranging from six months to six years. ${ }^{21}$ The authors described extensively the anatomy of the inguinal and femoral regions and adopted the principle of broad support against pressure, based on Pascal's law and minimal biological response using a lightweight mesh to cover the femoral ring, public tubercle, area of supra-vesical hernia and the lateral triangle of the inguinal canal. ${ }^{21}$ Workers from India and China share similar views on the anatomic and technical approaches. ${ }^{24}$

A correct pre-operative diagnosis of femoral hernia is a requisite condition for planning a meaningful operative strategy. In the current review, $29(22.7 \%)$ patients were not aware of any pre-existing groin swellings at the time of first contact with the surgeon, and of these, correct diagnosis was missed in 19 (65.5\%) patients leading to initial clinical error in diagnosis and delay. However, combined diagnostic information from abdominal imaging studies and clinical re-evaluation over the following hours and sometimes days allowed correct diagnoses to be made in the majority $(16,84.2 \%)$ of the 19 cases preoperatively, leaving only three $(15.8 \% \%)$ cases to be diagnosed intra-operatively. Similarly, at Los Angeles General County Hospital, 40\% of patients treated for strangulated femoral hernias were not aware of any herniation before presentation..$^{25}$ The author also argued that the 12 deaths that occurred among the remaining $60 \%$ who knew of their hernias before strangulation could have been prevented by elective repair of the hernias. ${ }^{25}$ 
Steven and Julie of Aberdeen, UK reported on a case series of five strangulated femoral hernias that were all missed at the time of initial clinical evaluation due to no evidence of external hernia to warrant the diagnosis. ${ }^{12}$ The delay in diagnosis of the hernias reported in the series was on the average 4.7 days which necessitated bowel resection in 60.0\% (3 cases) and over-sewing of a necrotic band due to Richter's type hernia in $40.0 \%$ (two case). ${ }^{12}$ From the above, femoral hernias especially the strangulated type, still contribute significant diagnostic pitfalls and every surgeon involved in treatment of femoral hernia must be aware of this common clinical challenge if the delays and bowel resections are to be minimized.

The bowel resection rate of $63.0 \%$ for emergency cases recorded in this study is clinically significant, but like previous authors have noted, delays in presentation and diagnosis were major reasons for the high rates of intestinal necrosis and gangrene. ${ }^{8,12,21}$ The report by Odula and Kakande from Kampala, Uganda ${ }^{18}$ that no cases of gangrene were noted among 10 emergency femoral hernia repairs was an unusual situation. However, the authors ${ }^{18}$ described an unusually high relationship between onchocerciasis and femoral hernia in West Nile district of Uganda, and probably femoral hernias with anatomic peculiarity not prone to strangulation may exist in Uganda. Further, more elaborate study may be required to clarify the above findings.

The use of general and spinal anesthesia in over threequarter $(79.7 \%)$ was perhaps, due to high rates of strangulated cases and associated large numbers of patients with ASA III and IV scores (Table 4). Typically, general and spinal anaesthesia offered better relaxation and longer time needed in this study, making them the most frequent technique compared to local infiltative anesthesia.

The post-operative complications recorded in this study are comparable to findings in previous studies. ${ }^{2,8,21}$ The wound infection rate of $31.3 \%$ was very high compared to a value of 4.3\% quoted in Cairo, Egypt. ${ }^{2}$ The explanation for the wide disparity may be due to the fact that the study in Egypt involved only uncomplicated femoral hernias which resulted in fewer wound complications. However, all but two wound infections in this series were managed conservatively with antibiotics and wound dressing. The overall recurrence rate $(5.5 \%)$ in this study is similar to a rate of $4.8 \%$ recorded by Milivoje and coworkers. ${ }^{8}$ Milivoje and coworker reported that only seven of the 83 patients managed for complicated femoral hernia had prosthetic mesh repair, which partly explained the high rates of recurrences observed during the 6-36 months follow up. In the complicated group in our series, no mesh was used, and combined with the higher infection rate may have contributed to the rate of recurrence observed in this study.

In this series, the high mortality raterecorded correlate with enormous delays that led to high rates of gangrene and bowel resections. Rogers reported on a series of 170 patients with strangulated femoral hernias and documented mortality rates of $11.4 \%$ and $13.0 \%$ among patients whose diagnoses were made pre-operatively and intra-operatively respectively. ${ }^{25} \mathrm{The}$ author further observed that deaths occurred in $8.3 \%$ of those who were diagnosed correctly within 24 hours of onset of complication compared to $47 \%$ in those made after 24 hours. ${ }^{25}$ A recent publication from Turkey, however, recorded lower mortality rate of $1.6 \%$. ${ }^{9}$ The prolonged delays before surgical interventions and the unusually high comorbid medical conditions in our series probably accounted for the higher mortality and prolonged hospital stay compared to the Turkish series ${ }^{9}$ with minimal delays and optimized comorbid states.

\section{Limitation}

This was a retrospective review and as such did not have a design that would thoroughly assess outcomes like wound infection, recurrence and mortality. The follow up was passive and relatively short and could not have captured the representative rates of the various outcomes. An elaborate cross-sectional analytical study is recommended.

\section{CONCLUSION}

This study generated the firstregional original article on femoral hernia in southeast Nigeria and the first multicenter research on the subject in Nigeria to the best of our knowledge. Femoral hernias are relatively uncommon, but have the high propensity towards strangulation. Efforts should be made to repair these hernias electively because of high morbidity and mortality associated with emergency operations. Precise preoperative diagnosis is imperative and surgeons who undertake repairs of femoral hernias must be conversant with the unusual manners the hernias can present, in order to overcome the diagnostic pitfalls and the delays in management.

\section{Key short messages}

1. Femoral hernia has the high propensity towards strangulation; thus, many patients presented emergently and have bowel resection.

2. Femoral hernia was more common in older patients who commonly harbored comorbidities; therefore, morbidity and mortality rates were high.

3. The rate of diagnostic pitfalls was high leading to missed diagnoses in many occasions.

4. The uptake of mesh for elective femoral hernia in our environment is low.

\section{ACKNOWLEDGEMENT}

We express our gratitude to the board of consultants, general surgery section of our institutions for their understanding and cooperation throughout the period of this study.

Asian Journal of Medical Sciences | Nov-Dec 2020 | Vol 11 | Issue 6 


\section{REFERENCES}

1. Sorelli PG, El-Masry NS and Gairett W. Open femoral hernia repair: one skin incision for all. World J Surg. 2009; 4:44.

https://doi.org/10.1186/1749-7922-4-44

2. Arafat M. Mesh Plug for treatment of femoral hernia. EJPMR. 2017; 4(10): 155-158.

3. Hachisuka T. Femoral hernia repair. Surg Clin N AM. 2003; 83:1189-1205.

https://doi.org/10.1016/S0039-6109(03)00120-8

4. Sucandy I and Kolff JW. Incarcerated femoral Hernia in Men: Incidence, Diagnosis and Surgical Management. NAM J Med Sci. 2012; 4(11): 617-618.

https://doi.org/10.4103/1947-2714.103343

5. Fitzgibbons RJ and Forse RA. Groin Hernias in Adults. N Engl J Med. 2015; 372(8):756-763.

https://doi.org/10.1056/NEJMcp1404068

6. Akrami M, Karami MY, Zangouri V, Deilami I and Maalhagh M. Small Bowel Obstruction Secondary to Femoral Hernia. Case Report and Review of the literature. Bull Emerg Trauma. 2016;4(1);51-53.

7. Ajao OG. Obstructed Groin Hernia in a Tropical African Population. J Nat Med Ass. 1979; 71(11):1093-1094.

8. Vukovic M, Moljevic N and Crnogorac S. Tension Free Femoral hernia repair with Plug. J Acute dis. 2013; 2013: 40-43.

https://doi.org/10.1016/S2221-6189(13)60093-1

9. Kunduz E, Sormaz IC, Yopalak Y, Bektasoglu HK and Gok AFK. Comparison of surgical techniques and results for emergency and elective femoral hernia repair. Ulus Trauma Acil Cerrahi Derg. 2019; 25(6);611-615.

https://doi.org/10.14744/tjtes.2019.04524

10. Frank G. Femoral Hernia in females. Canad Med Ass J. 1965; 93:1346-1350.

11. Nguyen ET and Komenaka IK. Strangulated Femoral hernia containing a perforated appendix. Can J Surg. 2004; 47(1): 68-69.

12. Heys SD and Brittenden J. Strangulated femoral hernia: the persistent clinical trap. Postgrad Med J. 1991; 67:57-59. https://doi.org/10.1136/pgmj.67.783.57

13. Dauda MM and Said NM. Giant Femoral Hernia in a Male. Arin Afr Med. 2004; 3(4):197-198.

14. Ursula D, Staffan W, Par N, Gabriel S and Ulf G. Emergency femoral Hernia. Repair: A study Based on a National Register. Ann Surg. 2009: 249(4):672-676.

https://doi.org/10.1097/SLA.0b013e31819ed943

15. Garba ES. The Pattern of Adult External Abdominal Hernias in Zaria. Nig J Surg Res. 2000; 2(1):12-15. https://doi.org/10.4314/njsr.v2i1.12176

16. Nikolopoulos I, Oderuth E, Ntakomyti E and Kald B. Intestinal obstruction due to bilateral strangulated femoral hernias. Case Rep Surg. 2014; 2014: el95736. https://doi.org/10.1155/2014/195736

17. Balram $M$ and Srivastava A. Prevalence of femoral Hernia in Bundelkhand region of India. Ann Int. Med Den Res. 2016: 2(6):38-40 https://doi.org/10.21276/aimdr.2016.2.6.SG10

18. Odula PO and Kakande I. Femoral Hernia at Mulago Hospital, Uganda. East Cent Afr J Surg. 2004; 9(2): 74-77.

19. Abi-Haidar $Y$, Sanche $Z V$ and Itani KM. Risk Factors and Outcomes of Acute Versus Elective Groin Hernia Surgery. J AM Coll Surg. 2011; 213(3):364-369. https://doi.org/10.1016/j.jamcollsurg.2011.05.008

20. Kumar M, Sinha KK and Ranjan R. Small Bowel Obstruction Secondary to Femoral Hernia. Int J Contemp Med Res. 2017; 4(2): 573-574.

21. Mike $M$ and Kano N. Femoral Hernia: A Review of the Clinical Anatomy and Surgical Treatment Surg Sci. 2013; 4: 453-548. https://doi.org/10.4236/ss.2013.410089

22. Mabula JB and Chalya PL. Surgical management of inguinal hernias at Bugando Medical Centre in Northwestern Tanzania: Our experiences in a resource-limitedsetting. BMC Res Notes. 2012;5:585.

https://doi.org/10.1186/1756-0500-5-585

23. Ogbuanya $A U$ and Emedike SOC. Elective repair of uncomplicated inguinal in southeast Nigeria. Asian Journal of Medical Sciences. 2015; 7(2): 90-95. https://doi.org/10.3126/ajms.v7i2.13349

24. Ram BR, Goud VS, Kumar DR, Reddy B K, Boda KS and Madipeddi V. Strangulated Groin Hernia Repair: A New Approach for all. J Clin Diag Res. 2016;10(4):04-06. https://doi.org/10.7860/JCDR/2016/18037.7613

25. Rogers FA. Strangulated femoral hernia: A review of 170 cases. Ann Surg. 1959; 149 (1):9-20. https://doi.org/10.1097/00000658-195901000-00002

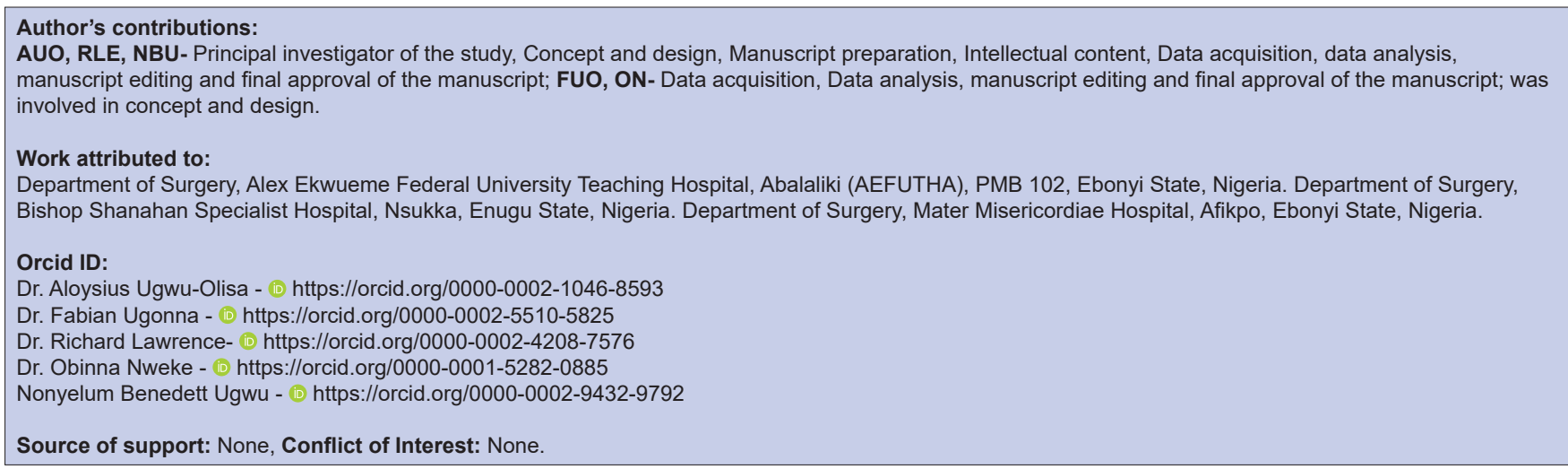

